\title{
Deteção Precoce de COVID-19 em Portugal: Uso de Registos Clínicos
}

\section{Early Detection of COVID-19 in Portugal: Use of Clinical Records}

\author{
Ana Rita TORRES $\otimes^{1}$, Susana SILVA ${ }^{1}$, Irina KISLAYA ${ }^{1,2,3}$, João Pedro MARTINS ${ }^{4}$, Carlos MATIAS DIAS ${ }^{1,2,3}$, \\ Ana Paula RODRIGUES ${ }^{1}$ \\ Acta Med Port 2021 Mar;34(3):176-184 - https://doi.org/10.20344/amp.14593
}

\section{RESUMO}

Introdução: A vigilância sindrómica permite a identificação precoce de alterações no padrão de morbilidade da população. Este estudo tem como objetivo avaliar a utilidade de indicadores relativos a cuidados de saúde primários e hospitalares, na vigilância da COVID-19. Material e Métodos: Foi realizada uma análise de séries temporais utilizando a taxa de incidência semanal de COVID-19 em Portugal Continental, entre as semanas 14/2020 (30 março a 05 abril) e 25/2020 (15 a 21 junho), e seis indicadores: 1) consultas em cuidados de saúde primários por COVID-19; 2) número de episódios de urgência por COVID-19; 3) número de episódios de urgência por pneumonia vírica; 4) número de internamentos por pneumonia vírica; 5) proporção de episódios de urgência por pneumonia vírica face ao total de episódios de urgência por pneumonia; e 6) proporção de internamentos por pneumonia vírica face ao total de internamentos por pneumonia. Foram calculadas correlações de Pearson e correlações cruzadas.

Resultados: Foi encontrada uma correlação forte entre a taxa de incidência semanal de COVID-19 e todos os indicadores [(1) 0,76 ; (2) 0,$82 ;(3) 0,77 ;(4) 0,84 ;(5) 0,86$; e (6) 0,90]. Os episódios de urgência e internamento por pneumonias víricas detetam variações na frequência da doença, com uma semana de antecedência. As consultas em cuidados de saúde primários e urgências por COVID-19 registam uma semana de atraso relativamente à evolução da taxa de incidência. A proporção de pneumonias víricas face ao número de pneumonias em episódios de urgência, ou internamentos, encontra-se alinhada temporalmente com a evolução da taxa de incidência semanal de COVID-19.

Discussão: O atraso encontrado no padrão de evolução de consultas em CSP, e de episódios de urgência por COVID-19 face à incidência de COVID-19, poderá estar relacionado com a reorganização dos serviços de saúde e criação de códigos específicos para estas consultas. Episódios de urgência e internamentos por pneumonia vírica poderão ser úteis para a deteção precoce de possíveis surtos de COVID-19. Pneumonias víricas poderão ter sido classificadas como pneumonias de causa indeterminada. A monitorização futura destes indicadores é necessária de modo a averiguar se a incidência de COVID-19 é influenciada significativamente por alterações na estratégia de testagem. Os indicadores deste trabalho serão uma mais valia para a adequação de estratégias de testagem, alocação de recursos de saúde a comunidades mais vulneráveis à morbilidade severa e avaliação de programas de vacinação. Como tal, os sistemas de vigilância com base em registos de saúde serão um complemento valioso ao SINAVE.

Conclusão: Sugere-se que os indicadores em análise sejam utilizados de forma regular, com especial atenção à informação relativa a pneumonias víricas, como forma de detetar precocemente surtos de COVID-19. A informação relativa a pneumonias de causa indeterminada poderá ser considerada na monitorização da COVID-19.

Palavras-chave: COVID-19; Infecções por Coronavírus/diagnóstico; Pneumonia Viral/diagnóstico; Portugal; SARS-CoV-2; Vigilância de Evento Sentinela

\section{ABSTRACT}

Introduction: Syndromic surveillance allows early detection of changes in the population's morbidity pattern. The aim of this study is to evaluate the usefulness of indicators related to access to healthcare services, in COVID-19 surveillance.

Material and Methods: A time series analysis was performed using the weekly incidence rate of COVID-19 in Mainland Portugal, between weeks 14/2020 (March 30 to April 5) and 25/2020 (June 15 to 21), and six indicators: 1) COVID-19 consultations in primary healthcare; 2) number of COVID-19 emergency department visits; 3) number of emergency department visits due to viral pneumonia; 4) number of hospitalizations due to viral pneumonia; 5) proportion of emergency department visits due to viral pneumonia; and 6) proportion of hospitalizations for viral pneumonia. Pearson correlation and cross-correlations were computed.

Results: A strong correlation was found between the weekly incidence rate of COVID-19 and all indicators. [(1) 0.76 ; (2) 0.82 ; (3) 0.77 ; (4) 0.84 ; (5) 0.86 ; e (6) 0.90 ]. Emergency department visits and hospitalizations for viral pneumonia detect variations in the frequency of the disease with a one week lag compared to the incidence rate of COVID-19, in one week. COVID-19 consultations in primary healthcare and emergency department visits trail behind the incidence rate of COVID-19, in one week. The proportion of viral pneumonias in emergency department visits, or hospitalizations, is temporally aligned with the weekly incidence rate of COVID-19.

Discussion: The delay found in the COVID-19 primary healthcare consultations and emergency department visits, may be related to changes in access to healthcare services and clinical coding. Emergency department visits and hospitalizations for viral pneumonia may be useful in the early detection of COVID-19. Viral pneumonia may have been coded as being of unknown origin. Future monitoring of these indicators is necessary to ascertain whether the incidence of COVID-19 is significantly influenced by changes in testing strategies. The indicators described in this study will be an asset for the optimization of testing strategies, allocation of healthcare resources

1. Departamento de Epidemiologia. Instituto Nacional de Saúde Doutor Ricardo Jorge. Lisboa. Portugal.

2. Centro de Investigação em Saúde Pública. Escola Nacional de Saúde Pública. Universidade NOVA de Lisboa. Lisboa. Portugal.

3. Comprehensive Health Research Center. Faculdade de Ciências Médicas de Lisboa. Lisboa. Portugal.

4. Advanced Analytics and Intelligence Unit. Direção Sistemas de informação. Serviços Partilhados do Ministério da Saúde. Lisboa. Portugal.

$\triangle$ Autor correspondente: Ana Rita Torres. a.rita.torres@insa.min-saude.pt

Recebido: 18 de julho de 2020 - Aceite: 13 de novembro de 2020 - Online issue published: 01 de março de 2021

Copyright @ Ordem dos Médicos 2021 
to the communities that are most vulnerable to severe morbidity and assessing vaccination impact. As such, surveillance systems based on clinical data will be a valuable complementary tool to SINAVE.

Conclusion: The indicators under analysis could be used regularly, with special attention to viral pneumonias, to detect outbreaks of COVID-19. Information on pneumonia of unknown etiology may be considered in the surveillance of COVID-19.

Keywords: COVID-19; Coronavirus Infections/diagnosis; Pneumonia, Viral/diagnosis; Portugal; SARS-CoV-2; Sentinel Surveillance

\section{INTRODUÇÃO}

A doença infeciosa causada pelo coronavírus da síndrome respiratória aguda grave 2 (SARS-CoV-2), à data designada por coronavirus disease 2019 ou COVID-19, foi declarada pela Organização Mundial de Saúde (OMS) como uma emergência de Saúde Pública de âmbito internacional no dia 30 de janeiro de 2020 e como pandemia no dia 11 de março. ${ }^{1}$

Desde o início da epidemia que Portugal, à semelhança dos demais países, deu prioridade aos trabalhos de preparação e resposta à doença provocada pelo novo Coronavírus, identificando três componentes principais de vigilância: verificação e deteção; avaliação do risco e gravidade; e a monitorização da epidemia. ${ }^{2}$ À data, em Portugal, a vigilância da COVID-19 assenta na identificação e notificação de casos de casos confirmados (tendo em conta a origem, apresentação clínica dos casos, destino do doente, contactos e localização do mesmo) através do Sistema Nacional de Vigilância Epidemiológica (SINAVE). A notificação de casos positivos é de carácter obrigatório, devendo ser registada nesta plataforma por médicos e laboratórios, conforme o publicado na Norma 004/2020 de 23/03/2020, atualizada em 25 de abril de $2020 .^{3}$

Contudo, conforme o preconizado pelo Plano Nacional de Preparação e Resposta à Doença por Novo Coronavírus, com o crescimento gradual da epidemia em Portugal, a partir da confirmação do primeiro caso de doença no dia 2 de março de 2020, a vigilância deveria deixar de assentar exclusivamente na identificação e notificação de todos os casos confirmados, devendo centrar-se em sistemas de vigilância sindrómica. ${ }^{2,4}$

Esta recomendação foi também difundida pelo Centro Europeu de Controlo de Doenças (ECDC) no âmbito do combate à pandemia de COVID-19, como forma de monitorizar a disseminação do vírus pela comunidade, avaliar a eficácia de medidas de contingência, ou a evolução da doença face ao relaxamento das mesmas. ${ }^{5} \mathrm{~A}$ eficácia dos sistemas de vigilância sindrómica, por oposição a outros sistemas de monitorização e vigilância em saúde, resulta do uso de informação que não necessita de confirmação laboratorial, ou que esteja diretamente dependente de profissionais de saúde como, por exemplo, dados de auto-reporte ou registos clínicos automatizados. Este procedimento permite mobilizar uma resposta mais rápida e, assim, reduzir o número de novas infeções e óbitos atempadamente. ${ }^{6}$

Estes objetivos ganham particular relevância se for tido em conta o tempo decorrido entre a infeção e a notificação da doença: estima-se que a vigilância laboratorial de COVID-19 revele o estado de evolução da doença na população, com duas a quatro semanas de atraso. ${ }^{7}$ Por outro lado, a existência de situações de subnotificação laboratorial ou de incongruências em bases de dados oficiais (decorrentes de alterações na estrutura de informação, de definição de caso, ou vieses na data de notificação) ${ }^{8}$ torna evidente a necessidade de complementar os sistemas de vigilância laboratorial com outros que permitam uma monitorização da doença mais precoce.

A vigilância sindrómica tem sido um meio de vigilância epidemiológica em doenças do trato respiratório, como a gripe e infeções respiratórias agudas. ${ }^{6,9-13}$ Uma vez que a apresentação sintomática da gripe é semelhante à da COVID-19, a pergunta que se segue surge naturalmente: será que os indicadores que poderiam ser utilizados para a monitorização da gripe, como o número de consultas em cuidados de saúde primários, ou internamentos por pneumonia, se poderão transpor com eficácia para a vigilância da COVID-19? $?^{11,14,15}$

Alguns estudos tentaram responder a esta questão, apesar do início recente da epidemia. No estado do Kentucky, um indicador de vigilância sindrómica composto, incorporando informação sobre episódios de urgência e internamentos hospitalares (por COVID-19 e gripe) foi utilizado para o processo de decisão em saúde pública, responsável pelo desconfinamento da região. ${ }^{16}$ Por sua vez, a plataforma Saúde Pública Inglaterra (Public Health England) utilizou indicadores relativos a consultas em cuidados de saúde primários e a episódios de urgência hospitalar (por COVID-19 e pneumonia) para a monitorização da COVID-19. ${ }^{17,18}$ Este sistema de vigilância contribuiu para a deteção do pico de incidência da primeira vaga de COVID-19 no Reino Unido. ${ }^{19}$

Em particular, variações no padrão de acesso a cuidados de saúde por pneumonias víricas podem indicar circulação do agente SARS-CoV-2 na comunidade, já que esta é uma das formas de apresentação da doença COVID-19. ${ }^{20,21}$ Porém, do nosso conhecimento, ainda não foi efetuado nenhum estudo para avaliar a eficácia destes indicadores na monitorização da COVID-19, em Portugal.

Deste modo, pretende-se avaliar a utilidade de indicadores sindrómicos (relativos à suspeita de doença por COVID-19) e indicadores relativos a diagnósticos relacionados com pneumonias na monitorização da COVID-19 em Portugal, comparando-os com a taxa de incidência semanal de COVID-19, no período compreendido entre as semanas 14/2020 (30 de março a 05 de abril) e 25/2020 (15 a 21 de junho). Para este fim, foram escolhidos dados relativos a cuidados de saúde primários e hospitalares, pela facilidade de obtenção da informação, pela eficácia demonstrada na monitorização da doença segundo a literatura relacionada, e pela experiência de uso de dados desta natureza na monitorização da gripe. ${ }^{11,14-16,19} \mathrm{O}$ início do período temporal de análise para este trabalho [semana 14/2020 (30 de março a 05 de abril)] foi escolhido tendo em conta o início da implementação de áreas dedicadas COVID-19 em cada serviço 
de Urgência (ADC-SU) e áreas dedicadas COVID-19 comunidade (ADC-COMUNIDADE) ${ }^{3} \mathrm{O}$ final do período temporal de análise [25/2020 (15 a 21 de junho)] prende-se com a informação mais atual disponível à data do estudo.

\section{MATERIAL E MÉTODOS}

Foi realizada uma análise de séries temporais utilizando a taxa de incidência semanal de COVID-19 em Portugal Continental, no período compreendido entre as semanas 14/2020 (30 de março a 05 de abril) e 25/2020 ( 15 a 21 de junho), e seis indicadores obtidos no universo de serviços públicos de saúde de Portugal Continental. Foram considerados os indicadores sindrómicos: (1) consultas em cuidados de saúde primários (CSP) por COVID-19; e (2) número de episódios de urgência por COVID-19. Foram ainda considerados os seguintes indicadores de diagnósticos relativos a pneumonias: (3) número de episódios de urgência por pneumonia vírica; (4) número de internamentos devido a pneumonia vírica; (5) proporção de episódios de urgência por pneumonia vírica face ao total de episódios de urgência por pneumonia (de qualquer etiologia); e (6) proporção de internamentos devido a pneumonia vírica face ao total de internamentos devido a pneumonia (de qualquer etiologia).

Por uma questão de uniformização, o período utilizado para a análise de séries temporais foi o mesmo em todos os indicadores. O limite inferior do período temporal foi escolhido tendo em conta o início da implementação de ADC-SU e ADC-COMUNIDADE, conforme a Norma $n^{\circ}$ 004/2020 de 23/03/2020, vigente desde as 00:00 horas do dia 26 de março de $2020 .^{3}$

A taxa de incidência semanal é expressa por 100.000 habitantes. Os restantes indicadores são expressos em números absolutos ou números relativos ao total de consultas em CSP ou ao total de internamentos, uma vez que não está disponível o denominador populacional para cálculo das taxas respetivas.

\section{Taxa de incidência de COVID-19}

A informação relativa aos casos de COVID-19, confirmados laboratorialmente pela rede de laboratórios que a nível nacional realiza o diagnóstico de COVID-19, é proveniente do SINAVE e foi atualizada a 26 de junho de 2020. Foram obtidas as contagens de casos confirmados de COVID-19 por semana, tendo como base a data de notificação de novas infeções em cada dia, e calculadas as taxas de incidência semanal utilizando as estimativas anuais da população residente em Portugal Continental para 2018 disponibilizadas pelo Instituto Nacional de Estatística. ${ }^{22}$

\section{Consultas por COVID-19 em Cuidados de Saúde Primá- rios}

Os dados relativos a consultas por COVID-19 em CSP foram disponibilizados pelos Serviços Partilhados do Ministério da Saúde, E.P.E. (SPMS) e atualizados a 26 de junho de 2020. A totalidade dos agrupamentos de centros de saúde (ACES) em Portugal Continental está abrangida por esta informação.
Foram considerados os códigos ICPC-2 A77.01 (infeção por COVID-19) e ICPC-2 A29.01 (suspeita de infeção por COVID-19), no âmbito das recomendações emitidas pelo Centro de Terminologias Clínicas (CTC) para o registo de informação clínica relacionada com a doença COVID-19 nos sistemas de informação da saúde em Portugal e que tiveram por base a classificação Internacional de Cuidados de Saúde Primários (ICPC-2). ${ }^{23}$ As contagens semanais foram obtidas com base na data de consulta.

Episódios registados como COVID-19, pneumonias víricas e pneumonias de qualquer etiologia em urgência hospitalar e internamento

Os dados relativos aos episódios de urgência por COVID-19, de pneumonias víricas e de pneumonia de qualquer etiologia (em urgência ou internamento), em serviços de saúde públicos, têm como base diagnósticos atribuídos administrativamente, tendo sido disponibilizados pela SPMS e atualizados a 26 de junho de 2020. A proporção de hospitais públicos e serviços básicos de urgência (SUB) cobertos pela informação relativa a episódios de urgência é $57,1 \%$. A proporção de hospitais públicos cobertos pela informação relativa a internamentos é $45,5 \%$. A listagem de hospitais públicos e serviços de urgência utilizados na compilação da informação manteve-se constante, pelo que ambas as proporções aqui reportadas não variaram durante o período em análise.

Para a contabilização dos episódios de urgência por COVID-19 foram considerados os códigos U07.1 (COVID-19, vírus identificado) e U07.2 (COVID-19, vírus não identificado), no âmbito das recomendações emitidas pelo CTC para o registo de informação clínica relacionada com a doença COVID-19 nos sistemas de informação da saúde em Portugal, e que tiveram por base a $10^{a}$ edição da Classificação Internacional de Doenças (CID-10) da OMS. ${ }^{23}$

Para a contabilização dos episódios de urgência e internamento hospitalar por pneumonia de qualquer etiologia foram considerados os códigos 480 a 486 da nona edição da Classificação Internacional de Doenças (CID-9) da OMS ou os códigos J12 a J18 da CID-10.

Para a contabilização dos episódios de urgência e internamento hospitalar por pneumonia vírica foram considerados os códigos 480 da CID-9 ou J12 da CID-10.

As contagens semanais foram obtidas com base na data do episódio de urgência ou internamento.

Foram incluídos nesta recolha de informação todos os hospitais de referência preparados para receber casos de COVID-19: Hospital de Braga, Hospital de São João, Hospital de Santo António, Hospital Pedro Hispano, Hospital Pediátrico de Coimbra, Hospital Sousa Martins, Hospital Nossa Senhora da Assunção, Hospital Curry Cabral, Hospital Egas Moniz, Hospital de São Francisco Xavier, Hospital de Dona Estefânia e Hospital de Faro.

\section{Análise estatística}

Foram calculadas correlações entre os seguintes indicadores: 
1. Taxa de incidência de COVID-19 semanal e número semanal de consultas por COVID-19 em CSP;

2. Taxa de incidência de COVID-19 semanal e número semanal de episódios de urgência por COVID-19;

3. Taxa de incidência de COVID-19 semanal e número semanal de episódios de urgência por pneumonia vírica;

4. Taxa de incidência de COVID-19 semanal e número semanal de internamentos por pneumonia vírica;

5. Taxa de incidência de COVID-19 semanal e proporção semanal de episódios de urgência por pneumonia vírica face ao total de episódios de urgência por pneumonia (de qualquer etiologia);

6. Taxa de incidência de COVID-19 semanal e proporção semanal de internamentos por pneumonia vírica face ao total de internamentos por pneumonia (de qualquer etiologia).

Inicialmente foi avaliada a relação linear entre os indicadores através do cálculo de correlações de Pearson $(0 \leq$ $p<0,3$ - correlação desprezível; $0,3 \leq p<0,5$ - correlação fraca; $0,5 \leq p<0,7$ - correlação moderada; $0,7 \leq p<0,9$ correlação forte; $0,9 \leq p$ - correlação muito forte). ${ }^{24}$ Numa segunda fase, foram calculadas as correlações cruzadas entre os mesmos indicadores.

O estudo de correlação cruzada permite a identificação do desfasamento (lag) entre duas séries temporais. Se o valor máximo de correlação cruzada ocorrer em zero, os valores da primeira série (taxa de incidência de COVID-19) estarão correlacionados com os valores da segunda série (restantes indicadores acima referidos), sem desfasamento. Se o valor de correlação cruzada mais alto tiver um lag negativo, os valores da primeira série serão correlacionados com os valores da segunda série com um atraso de lag semanas. Se pelo contrário, o valor de correlação cruzada mais elevado tiver um lag positivo, a segunda série precede a primeira em lag semanas. ${ }^{25}$

Todas as análises foram realizadas utilizando o programa $R$ Statistical Computing Enviroment. ${ }^{26}$

Este estudo não envolve participantes nem recolha ou tratamento de dados pessoais, de saúde, ou genéticos. Como tal, o Regime Geral de Proteção de Dados (RGPD) não é aplicável e não foi necessária aprovação por parte de Comissão de Ética do Instituto Nacional de Saúde, de acordo com a declaração de Helsínquia.

\section{RESULTADOS}

A Tabela 1 expressa a caraterização da taxa de incidência de COVID-19 e dos seis indicadores em análise, no período compreendido entre as semanas 14/2020 (30 de março a 05 de abril) e 25/2020 (15 a 21 de junho). O número semanal mais elevado de pneumonias víricas, quer em episódios de urgência quer em contexto de internamento hospitalar, foi registado uma semana antes do valor máximo observado para a taxa de incidência semanal de COVID-19.

A evolução da taxa de incidência semanal de COVID-19 face às consultas de CSP por COVID-19, episódios de urgência por COVID-19, episódios de urgência por pneumonia vírica e internamentos por pneumonia vírica pode ser consultada na Fig. 1. Apesar da tendência destes indicadores seguir um padrão semelhante, a evolução das consultas de CSP por COVID-19 (Fig. 1A) e episódios de urgência por COVID-19 (Fig. 1B) parece indicar um ligeiro atraso relativamente à taxa de incidência de COVID-19. Por outro lado, quer os episódios de urgência (Fig. 1C), quer os internamentos por pneumonia vírica (Fig. 1D), parecem preceder variações na taxa de incidência de COVID-19. As análises visuais foram confirmadas pelos valores de correlação cruzada apresentados na Tabela 2.

Foi observada uma correlação forte $(\rho>0,7)$ entre a taxa de incidência de COVID-19 e os indicadores em análise: 1) número de consultas de CSP por COVID-19; 2) número de episódios de urgência por COVID-19; 3) número de episódios de urgência por pneumonia vírica; 4) número de internamentos por pneumonia vírica; 5) proporção de episódios de urgência por pneumonia vírica face ao total de episódios de urgência por pneumonia (de qualquer etiologia); e 6) proporção de internamentos devido a pneumonia vírica face ao total de internamentos devido a pneumonia (de qualquer etiologia) (Tabela 3).

Os valores de correlação cruzada entre a taxa de incidência de COVID-19 e o número de consultas de CSP, bem como a correlação cruzada entre a taxa de incidência de COVID-19 e o número de episódios de urgência por

Tabela 1 - Caracterização da taxa de incidência de COVID-19 (100 000 habitantes) e seis indicadores em análise. Portugal Continental, semanas 14/2020 (30 de março a 05 de abril) a 25/2020 (15 a 21 de junho).

\begin{tabular}{|c|c|c|c|c|c|c|c|}
\hline & $\begin{array}{c}\text { Taxa } \\
\text { incidência } \\
\text { COVID-19 } \\
\text { (100 000 } \\
\text { habitantes) }\end{array}$ & $\begin{array}{l}\text { Consultas } \\
\text { CSP } \\
\text { CoVID-19 } \\
\text { (N) }\end{array}$ & $\begin{array}{l}\text { Episódios } \\
\text { urgência } \\
\text { COVID-19 } \\
\text { (N) }\end{array}$ & $\begin{array}{l}\text { Episódios } \\
\text { urgência } \\
\text { pneumonia } \\
\text { vírica } \\
\text { (N) }\end{array}$ & $\begin{array}{l}\text { Internamentos } \\
\text { pneumonia vírica } \\
(\mathrm{N})\end{array}$ & $\begin{array}{c}\text { Episódios } \\
\text { urgência } \\
\text { pneumonia } \\
\text { vírica } \\
/ \\
\text { total episódios } \\
\text { urgência } \\
\text { pneumonia } \\
(\%)\end{array}$ & $\begin{array}{c}\text { Internamentos } \\
\text { pneumonia vírica } \\
/ \\
\text { total internamentos } \\
\text { pneumonia } \\
(\%)\end{array}$ \\
\hline $\begin{array}{c}\text { Mínimo } \\
\text { (Ano/Semana) }\end{array}$ & $\begin{array}{c}18,35 \\
(2020 / 21)\end{array}$ & $\begin{array}{c}11940 \\
(2020 / 24)\end{array}$ & $\begin{array}{c}171 \\
(2020 / 24)\end{array}$ & $\begin{array}{c}10 \\
(2020 / 23)\end{array}$ & $\begin{array}{c}0 \\
(2020 / 19 \text { e } 2020 / 25)\end{array}$ & $\begin{array}{c}2,87 \\
(2020 / 24)\end{array}$ & $\begin{array}{c}0,00 \\
(2020 / 19 \text { e } 2020 / 25)\end{array}$ \\
\hline $\begin{array}{c}\text { Máximo } \\
\text { (Ano/Semana) }\end{array}$ & $\begin{array}{c}44,47 \\
(2020 / 15)\end{array}$ & $\begin{array}{c}48543 \\
(2020 / 16)\end{array}$ & $\begin{array}{c}668 \\
(2020 / 17)\end{array}$ & $\begin{array}{c}209 \\
(2020 / 14)\end{array}$ & $\begin{array}{c}61 \\
(2020 / 14)\end{array}$ & $\begin{array}{c}24,88 \\
(2020 / 14)\end{array}$ & $\begin{array}{c}45,19 \\
(2020 / 14)\end{array}$ \\
\hline
\end{tabular}




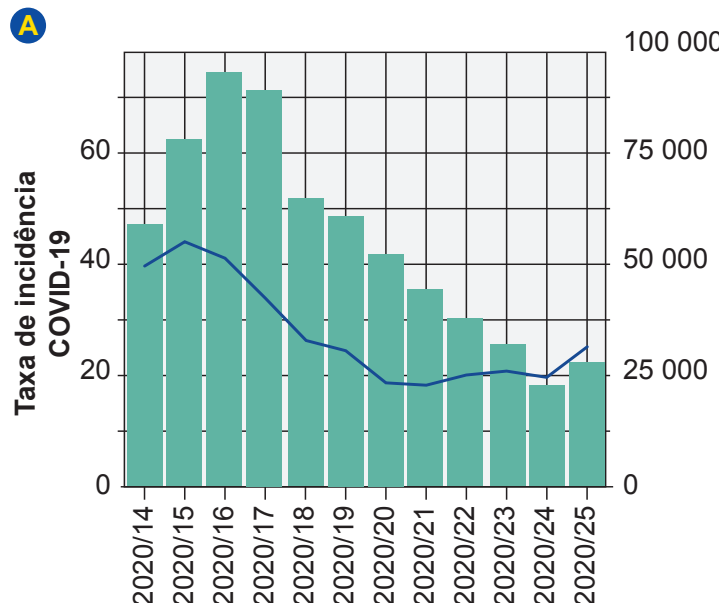

Ano/semana

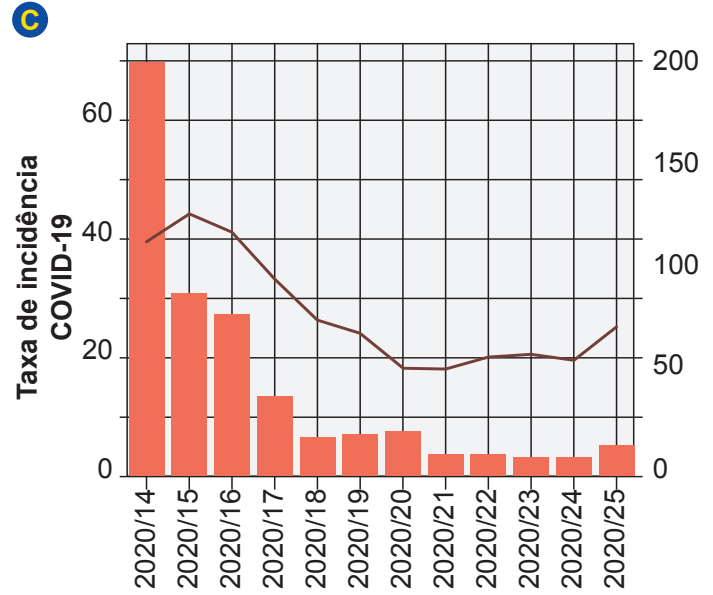

Ano/semana

E

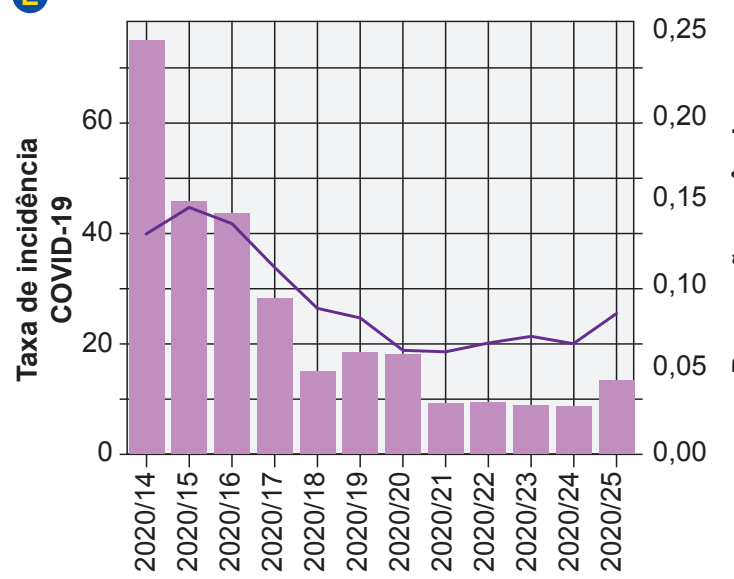

Ano/semana

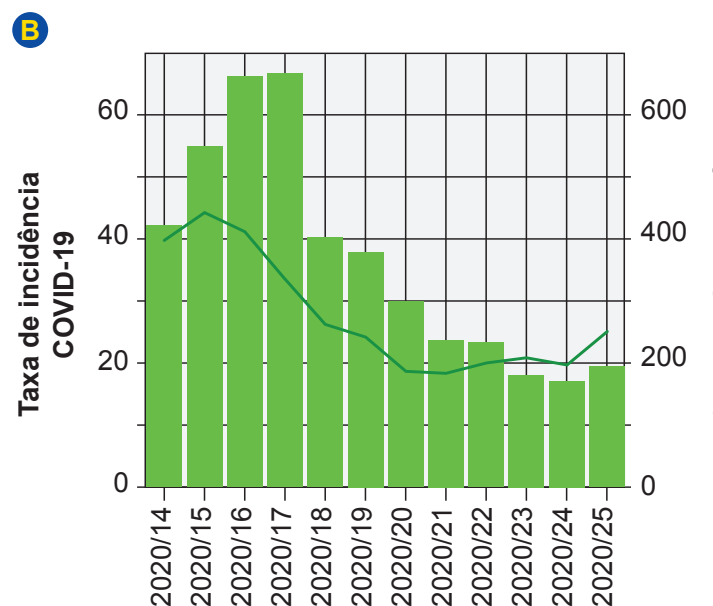

Ano/semana

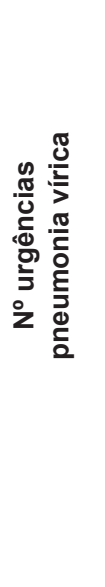

(D)
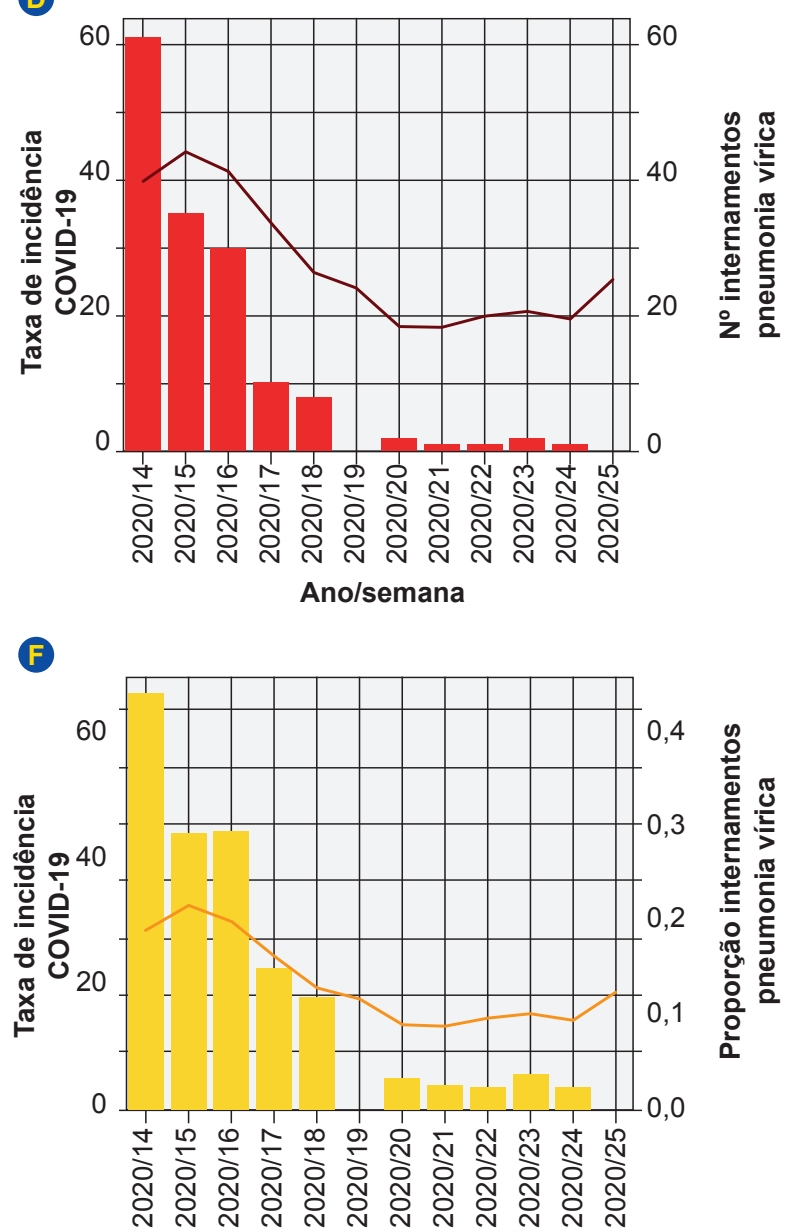

Ano/semana

Figura 1 - (A) Evolução semanal da taxa de incidência de COVID-19 em Portugal Continental (linha) e do número de consultas em cuidados de saúde primários (CSP) por COVID-19 (barra); (B) Evolução semanal da taxa de incidência de COVID-19 em Portugal Continental (linha) e do número de episódios de urgência por COVID-19 (barra); (C) Evolução semanal da taxa de incidência de COVID-19 em Portugal Continental (linha) e do número de episódios de urgência por pneumonia vírica (barra); (D) Evolução semanal da taxa de incidência de COVID-19 em Portugal Continental (linha) e do número de internamentos por pneumonia vírica (barra); (E) Evolução semanal da taxa de incidência de COVID-19 em Portugal Continental (linha) e da proporção de episódios de urgência por pneumonia vírica face ao total de episódios de urgência por pneumonia (de qualquer etiologia) (barra); (F) Evolução semanal da taxa de incidência de COVID-19 em Portugal Continental (linha) e da proporção de internamentos por pneumonia vírica face ao total de internamentos por pneumonia (de qualquer etiologia) (barra). 
COVID-19, revelaram que a taxa de incidência precede estes indicadores em cerca de uma semana (Tabela 2). Contudo, os valores de correlação cruzada entre a taxa de incidência por COVID-19, e o número de episódios de urgência por pneumonia vírica, bem como a correlação cruzada entre a taxa de incidência por COVID-19 e o número de internamentos por pneumonia vírica, revelam que estes indicadores precedem a taxa de incidência de COVID-19 em cerca de uma semana (Tabela 2). Tanto a proporção de episódios de urgência por pneumonia vírica face ao total de episódios de urgência por pneumonia, como a proporção de internamentos devido a pneumonia vírica face ao total de internamentos devido a pneumonia, apresentam valores de correlação cruzada superiores para lag $=0$, o que indica que estes indicadores se encontram alinhados temporalmente com a evolução da taxa de incidência e COVID-19 (Tabela 2).

\section{DISCUSSÃo}

O padrão de evolução de consultas de CSP e de episó- dios de urgência por COVID-19 é concordante com a evolução da taxa de incidência semanal de COVID-19, embora estes indicadores registem um atraso de cerca de uma semana relativamente à evolução da doença na comunidade. Esta dilação poderá estar relacionada com a reorganização dos serviços de saúde no início da epidemia: uma vez que as ADC-COMUNIDADE e ADC-SU foram sendo abertas, faseadamente, no início da pandemia, e poderia existir procura não correspondida, nomeadamente, a nível de consultas de CSP por COVID-19. Saliente-se, contudo, que a abertura das ADC-COMUNIDADE e ADC-SU foi pensada numa ótica preventiva e de modo a acautelar um aumento substancial do número de casos de COVID-19, tendo sido reforçada a sua oferta até à verificação da tendência decrescente da doença. Nesta perspetiva, espera-se que, no período em análise, as áreas dedicadas COVID-19 tenham conseguido dar resposta a todos os casos de COVID-19 que procuraram cuidados de saúde, mesmo que com atraso no seu atendimento, o que é uma hipótese consistente com o padrão de evolução dos indicadores.

Tabela 2 - Coeficientes de correlação cruzada entre a taxa de incidência de COVID-19 (por 100000 habitantes) e seis indicadores em análise. Portugal Continental, semanas 14/2020 (30 de março a 05 de abril) a 25/2020 (15 a 21 de junho).

\begin{tabular}{|c|c|c|c|c|c|c|}
\hline Lag & $\begin{array}{l}\text { Consultas CSP } \\
\text { COVID-19 } \\
(\mathrm{N})\end{array}$ & $\begin{array}{l}\text { Episódios urgência } \\
\text { COVID-19 } \\
\text { (N) }\end{array}$ & $\begin{array}{l}\text { Episódios urgência } \\
\text { pneumonia vírica } \\
(\mathrm{N})\end{array}$ & $\begin{array}{l}\text { Internamentos } \\
\text { pneumonia vírica } \\
(\mathrm{N})\end{array}$ & $\begin{array}{c}\text { Episódios urgência } \\
\text { pneumonia vírica } \\
\text { / } \\
\text { total episódios } \\
\text { urgência } \\
\text { pneumonia } \\
(\%)\end{array}$ & $\begin{array}{c}\text { Internamentos } \\
\text { pneumonia vírica } \\
\text { / } \\
\text { total internamentos } \\
\text { pneumonia } \\
(\%)\end{array}$ \\
\hline-11 & $-0,13$ & $-0,11$ & $-0,06$ & $-0,07$ & $-0,06$ & $-0,09$ \\
\hline-10 & $-0,34$ & $-0,28$ & $-0,15$ & $-0,17$ & $-0,17$ & $-0,21$ \\
\hline-9 & $-0,48$ & $-0,41$ & $-0,23$ & $-0,24$ & $-0,28$ & $-0,28$ \\
\hline-8 & $-0,49$ & $-0,44$ & $-0,27$ & $-0,27$ & $-0,33$ & $-0,30$ \\
\hline-7 & $-0,37$ & $-0,38$ & $-0,27$ & $-0,26$ & $-0,34$ & $-0,28$ \\
\hline-6 & $-0,19$ & $-0,27$ & $-0,22$ & $-0,24$ & $-0,27$ & $-0,25$ \\
\hline-5 & 0,06 & $-0,05$ & $-0,14$ & $-0,19$ & $-0,14$ & $-0,21$ \\
\hline-4 & 0,31 & 0,17 & $-0,07$ & $-0,10$ & $-0,03$ & $-0,09$ \\
\hline-3 & 0,58 & 0,50 & 0,02 & 0,01 & 0,10 & 0,08 \\
\hline-2 & 0,83 & 0,81 & 0,19 & 0,23 & 0,31 & 0,36 \\
\hline-1 & $0,94^{*}$ & $0,96^{*}$ & 0,40 & 0,50 & 0,56 & 0,64 \\
\hline 0 & 0,76 & 0,82 & 0,77 & 0,84 & $0,86^{*}$ & $0,90^{*}$ \\
\hline 1 & 0,39 & 0,47 & $0,81^{*}$ & $0,85^{*}$ & 0,82 & 0,84 \\
\hline 2 & 0,07 & 0,15 & 0,60 & 0,60 & 0,57 & 0,55 \\
\hline 3 & $-0,18$ & $-0,12$ & 0,29 & 0,27 & 0,24 & 0,22 \\
\hline 4 & $-0,33$ & $-0,30$ & 0,00 & $-0,02$ & $-0,07$ & $-0,07$ \\
\hline 5 & $-0,38$ & $-0,38$ & $-0,14$ & $-0,17$ & $-0,21$ & $-0,22$ \\
\hline 6 & $-0,35$ & $-0,36$ & $-0,31$ & $-0,34$ & $-0,34$ & $-0,38$ \\
\hline 7 & $-0,31$ & $-0,34$ & $-0,33$ & $-0,35$ & $-0,36$ & $-0,38$ \\
\hline 8 & $-0,23$ & $-0,25$ & $-0,30$ & $-0,33$ & $-0,32$ & $-0,34$ \\
\hline 9 & $-0,12$ & $-0,13$ & $-0,26$ & $-0,27$ & $-0,26$ & $-0,26$ \\
\hline 10 & $-0,03$ & $-0,05$ & $-0,23$ & $-0,22$ & $-0,21$ & $-0,20$ \\
\hline 11 & 0,00 & $-0,01$ & $-0,06$ & $-0,06$ & $-0,06$ & $-0,05$ \\
\hline
\end{tabular}

*A negrito, encontram-se assinalados os valores de correlação cruzada mais elevados. 
Tabela 3 - Correlação de Pearson entre a taxa de incidência de COVID-19 (por 100000 habitantes) e seis indicadores em análise. Portugal Continental, semanas 14/2020 (30 de março a 05 de abril) a 25/2020 (15 a 21 de junho).

\begin{tabular}{|c|c|c|c|c|c|c|}
\hline & $\begin{array}{l}\text { Consultas CSP } \\
\text { COVID-19 } \\
\text { (N) }\end{array}$ & $\begin{array}{l}\text { Episódios } \\
\text { urgência } \\
\text { COVID-19 } \\
\text { (N) }\end{array}$ & $\begin{array}{c}\text { Episódios } \\
\text { urgência } \\
\text { pneumonia vírica } \\
\text { (N) }\end{array}$ & $\begin{array}{l}\text { Internamentos } \\
\text { pneumonia vírica } \\
(\mathrm{N})\end{array}$ & $\begin{array}{c}\text { Episódios } \\
\text { urgência } \\
\text { pneumonia } \\
\text { vírica } \\
\text { / } \\
\text { total episódios } \\
\text { urgência } \\
\text { pneumonia } \\
(\%)\end{array}$ & $\begin{array}{c}\text { Internamentos } \\
\text { pneumonia vírica } \\
\text { / } \\
\text { total } \\
\text { internamentos } \\
\text { pneumonia } \\
(\%)\end{array}$ \\
\hline $\begin{array}{c}\text { Taxa de } \\
\text { incidência de } \\
\text { COVID-19 }\end{array}$ & $0,76^{*}$ & $0,82^{*}$ & $0,77^{*}$ & $0,84^{\text {** }}$ & $0,86^{* *}$ & $0,90^{\text {** }}$ \\
\hline
\end{tabular}

* significância a um nível de 99\%; ** significância a um nível de 99,9\%.

Outro fator a considerar para justificar este atraso prende-se com o momento em que foram criados e disponibilizados os códigos para estes motivos de consulta e episódios de urgência. Embora os códigos de registo de consultas e episódios de urgência com motivo COVID-19 tenham ficados disponíveis ainda antes da criação das áreas dedicadas COVID-19 (os primeiros registos datam de 17 de março de 2020), pode ter existido um período de transição na utilização dos mesmos. Por se tratar de uma doença cujos sintomas são semelhantes aos que estão associados à infeção pelo vírus Influenza e por outros vírus respiratórios sazonais, parte dos utentes que acederam a serviços de saúde em CSP, ou urgências hospitalares, em março, podem ter sido registados como tendo acedido a consultas e urgências por síndrome gripal.

O padrão de evolução de episódios de urgência e internamentos por pneumonia vírica é concordante com a evolução da taxa de incidência semanal de COVID-19. Verifica-se que variações nos episódios de urgência e internamentos por pneumonia vírica precedem variações na taxa de incidência de COVID-19, o que parece indicar que estes indicadores poderão ser úteis para a deteção precoce de possíveis surtos de COVID-19.

A evolução da proporção de pneumonias víricas face ao total de pneumonias em episódios de urgência e internamentos por pneumonia é concordante com o comportamento da taxa de incidência semanal de COVID-19, sem qualquer desfasamento temporal. Uma vez que seria expetável encontrar um padrão análogo ao observado para os indicadores anteriores (precedência face a variações na incidência de COVID-19), coloca-se como hipótese que existissem pneumonias víricas classificadas como sendo de causa indeterminada no período em análise, influenciando assim o seu valor relativo.

Não nos é possível, também, excluir a hipótese de que as codificações atribuídas a episódios de urgência ou internamento por pneumonia vírica, tenham sido menos usadas após a criação dos códigos associados à COVID-19, o que pode contribuir para a redução do número de registos destes episódios nas fases mais avançadas da epidemia. Consequentemente, e dado que se trata de um problema de saúde novo, vemos como necessário acompanhar a evolução destes indicadores, de forma a observarmos o seu comportamento em eventuais ondas epidémicas futuras. Esperamos, contudo, que um maior seguimento temporal aumente a força das associações encontradas, o que subsequentemente melhorará a deteção de variações na incidência de COVID-19.

Este seguimento é especialmente necessário para averiguar se alterações na estratégia de aplicação de testes de diagnóstico ao longo do período temporal em análise neste trabalho influenciaram de forma significativa a evolução da taxa de incidência de COVID-19 (por exemplo, através da priorização de testagem de pessoas sintomáticas no início da epidemia - Norma 004/2020 de 23/03/2020, atualizada em 25 de abril de 2020 - para uma estratégia de rastreio mais intensivo no final de maio, sobretudo em determinados setores de atividade profissional). ${ }^{3}$

A monitorização de COVID-19 exclusivamente por sistemas de vigilância fortemente dependentes de testagem pode não ser o mais indicado, sobretudo porque estes sistemas se encontrarão sempre limitados pela disponibilidade de kits de testagem e reagentes para a sua realização. Os indicadores utilizados neste trabalho, para além de não terem limitações de ordem logística, não são afetados de forma significativa por variações da definição de caso e estratégias de testagem, em especial nas pneumonias.

Os indicadores agora analisados têm uma correlação forte com a taxa de incidência de COVID-19, sendo, por isso, úteis à decisão em saúde pública, particularmente na adequação de estratégias de testagem e alocação de recursos de saúde a comunidades mais vulneráveis à morbilidade severa causada pela COVID-19. O facto de os indicadores referentes a pneumonias víricas poderem antecipar a deteção de variações significativas da frequência de infeção na população sugere que estes serão especialmente indicados para o direcionamento da estratégia de testagem como forma de identificar e isolar novos casos de infeção, prevenindo assim o aparecimento de novos surtos.

Adicionalmente, a vigilância sindrómica já demonstrou ser um aliado importante na avaliação da introdução de programas de vacinação. ${ }^{27,28}$ Por conseguinte, se as vacinas contra a COVID-19 forem distribuídas de forma ampla à população portuguesa, os sistemas de vigilância sindrómica podem desempenhar um papel essencial na avaliação do seu impacto na saúde da comunidade. 
Finalmente, a utilização de vários sistemas de vigilância com resultados concordantes reforça a evidência dos achados. Como tal, os sistemas de vigilância que fazem uso de registos clínicos para a monitorização da COVID-19 serão um complemento valioso ao atual sistema de vigilância universal em Portugal para a COVID-19, integrado no SINAVE. Note-se, ainda, que em situações de sobrecarga dos sistemas de vigilância epidemiológica pode haver maior subnotificação de casos, assumindo maior relevância a deteção de sinais em sistemas de vigilância com base em registos de saúde.

Consequentemente, se a confiança num sistema de vigilância construído com base nos indicadores agora em análise for elevada, o mesmo poderá ser uma mais valia na implementação de medidas de combate à epidemia de COVID-19. No âmbito deste sistema de vigilância, a deteção de um sinal que se venha a revelar falso positivo, poderá levar, todavia, ao desperdício de recursos (por exemplo, na validação do referido sinal). Contudo, uma vez que o sistema integra vários indicadores complementares, prevê-se que os mesmos se validem entre si, ou se validem com indicadores provenientes de outros sistemas e fontes de informação, minimizando este risco.

A finalidade do sistema de vigilância construído com os indicadores em análise é identificar um sinal precoce que nos possa dar um alerta do aumento da incidência de COVID-19, e não obter um modelo preditivo, pelo que não é imperativo anular o efeito de outros indicadores externos para confirmar se a relação entre os indicadores de vigilância e incidência de COVID-19 se mantém. Consequentemente, os sinais identificados continuam a ser relevantes, independentemente das relações de causalidade que possam vir a ser identificadas.

\section{REFERÊNCIAS}

1. World Health Organization. WHO Timeline - COVID-19. 2020. [consultado 2020 mai 03]. Disponível em: https://www.who.int/newsroom/detail/27-04-2020-who-timeline---covid-19.

2. Direção-Geral da Saúde. Plano Nacional de Preparação e Resposta à Doença Por Novo Coronavírus (COVID-19). Lisboa: DGS; 2020.

3. Direção-Geral da Saúde. Norma COVID-19: Fase de Mitigação. Portugal. Lisboa: DGS; 2020.

4. Direção-Geral da Saúde. Comunicado C160_75_v1. Casos de Infeção Por Novo Coronavírus (COVID-19). Lisboa: DGS; 2020.

5. European Centre for Disease Prevention and Control. Rapid risk assessment: coronavirus disease 2019 (COVID-19) in the EU/EEA and the UK-ninth update. Stockholm: ECDC; 2020.

6. Lazarus R, Kleinman KP, Dashevsky I, Demaria A, Platt R. Using automated medical records for rapid identification of illness syndromes (syndromic surveillance): the example of lower respiratory infection. BMC Public Health. 2001;1:9.

7. Güemes A, Ray S, Aboumerhi K, Desjardins M, Kvit A, Corrigan A, et al. A syndromic surveillance tool to detect anomalous clusters of COVID-19 symptoms in the United States. medRxiv. doi: 10.1101/2020.08.18.20177295.

8. Ashofteh A, Bravo J. A study on the quality of novel coronavirus (COVID-19) official datasets. Stat J IAOS. 2020;36:291-301.

9. Patterson-Lomba O, Van Noort S, Cowling B, Wallinga J, Gomes $M$, Lipsitch $M$, et al. Practice of epidemiology utilizing syndromic surveillance data for estimating levels of influenza circulation. Am J Epidemiol. 2014;179:1394-401.

\section{CONCLUSÃO}

Os indicadores analisados têm uma correlação forte com a taxa de incidência de COVID-19, sendo por isso úteis à decisão em saúde pública. Em particular, verifica-se que variações nos episódios de urgência e internamentos por pneumonia vírica precedem variações na taxa de incidência de COVID-19, o que parece indicar que estes indicadores poderão ser úteis para a deteção precoce de possíveis surtos de COVID-19 na população portuguesa. Em virtude de se colocar a hipótese de que existam pneumonias víricas classificadas como pneumonias de causa indeterminada vê-se como necessário avaliar a inclusão de um indicador composto, com informação relativa a pneumonias víricas e de causa indeterminada, na monitorização da COVID-19.

\section{PROTEÇÃO DE PESSOAS E ANIMAIS}

Os autores declaram que os procedimentos seguidos estavam de acordo com os regulamentos estabelecidos pelos responsáveis da Comissão de Investigação Clínica e Ética e de acordo com a Declaração de Helsínquia da Associação Médica Mundial atualizada em 2013.

\section{CONFIDENCIALIDADE DOS DADOS}

Os autores declaram ter seguido os protocolos do seu centro de trabalho acerca da publicação de dados.

\section{CONFLITOS DE INTERESSE}

Os autores declaram não ter conflitos de interesses relacionados com o presente trabalho.

\section{FONTES DE FINANCIAMENTO}

Este trabalho não recebeu qualquer tipo de suporte financeiro de nenhuma entidade no domínio público ou privado.

10. Harcourt S, Smith G, Elliot A, Pebody R, Charlett A, Ibbotson S, et al. Use of a large general practice syndromic surveillance system to monitor the progress of the influenza A (H1N1) pandemic 2009 in the UK. Epidemiol Infect. 2012;140:100-5.

11. Rosália P, Rodrigues AP, Silva S, Nunes B, Carlos M. Comparison between influenza coded primary care consultations and national influenza incidence obtained by the General Practitioners Sentinel Network in Portugal from 2012 to 2017. PLoS One. 2018;13:1-10.

12. Izquierdo J, Rus A, Jäger E, Buades A, Castell M, Fiol A, et al. Atención primaria vigilancia sindrómica de la gripe en atención primaria, un instrumento complementario a las redes centinelas para períodos de elevada incidencia de gripe. Aten Primaria. 2012;44:258-64.

13. Buehler J, Sonricker A, Paladini M, Soper P, Mostashari F. Syndromic surveillance practice in the United States: findings from a survey of state, territorial, and selected local health departments. Adv Dis Surveill. 2008;6:1-20.

14. McBean A, Hebert P. New estimates of influenza-related pneumonia and influenza hospitalizations among the elderly. Int J Infect Dis. 2004;8:22735.

15. Van den Wijngaard C, Van Asten L, Van Pelt W, Nagelkerke, N, Verheij R, De Neeling A, et al. Validation of syndromic surveillance for respiratory pathogen activity. Emerg Infect Dis. 2008;14:917-25.

16. Varela K, Scott B, Prather J, Blau E, Rock P, Vaughan A, et al. Primary indicators to systematically monitor COVID-19 mitigation and response Kentucky, May 19 - July 15, 2020. Morb Mortal Wkly Rep. 2020;69:11736. 
17. Public Health England. GP in Hours: Weekly Bulletins for 2020 2020. [consultado 2020 mai 03]. Disponível em: https://www.gov.uk/ government/publications/gp-in-hours-weekly-bulletins-for-2020.

18. Public Health England. Emergency Department Bulletin: 10 September 2020 Week 36. 2020. [consultado 2020 mai 03]. Disponível em: https:// assets.publishing.service.gov.uk/government/uploads/system/uploads/ attachment_data/file/916481/EDSSSBulletin2020wk36.pdf.

19. Public Health England. National COVID-19 surveillance reports 2020. [consultado 2020 jul 17]. Disponível em: https://www.gov.uk/ government/publications/national-covid-\%0A19-surveillance-reports.

20. Huang C, Wang Y, Li X, Ren L, Zhao J, Hu Y, et al. Clinical features of patients infected with 2019 novel coronavirus in Wuhan, China. Lancet. 2020;395:497-506.

21. Chan J, Yuan S, Kok K, To K, Chu H, Yang J, et al. A familial cluster of pneumonia associated with the 2019 novel coronavirus indicating person-to-person transmission: a study of a family cluster. Lancet. 2020;395:514-23.

22. Instituto Nacional de Estatística. Estimativas anuais da população residente. [consultado 2020 mai 15]. Disponível em: https://www.ine.pt/ xportal/xmain?xpid=INE\&xpgid=ine_indicadores\&contecto=pi\&indOcorr
Cod $=0008273 \&$ selTab=tab0\&xlang $=p t$.

23. Centro de Terminologias Clínicas. Normalização de Registos Inerentes à COVID-19. Lisboa: CTC; 202.

24. Mukaka MM. Statistics corner: a guide to appropriate use of correlation coefficient in medical research. Malawi Med J. 2012;24:69-71.

25. Montgomery D, Jennings C, Kulahci M. Introduction to time series analysis and forecasting. New Jersey: John Wiley \& Sons; 2015.

26. Foundation for Statistical Computing. A language and environment for statistical computing. Vienna, Austria. 2017. [consultado 2020 maio 03]. Disponivel em: https://www.r-project.org/.

27. Bawa Z, Elliot A, Morbey R, Ladhani S, Cunliffe N, O'Brien S, et al. Assessing the likely impact of a rotavirus vaccination program in England: the contribution of syndromic surveillance. Clin Infect Dis. 2015;61:77-85.

28. Pebody R, Sinnathamby M, Warburton F, Andrews N, Boddington $\mathrm{N}$, Zhao $\mathrm{H}$, et al. Uptake and impact of vaccinating primary schoolage children against influenza: experiences of a live attenuated influenza vaccine programme, England, 2015/16. Eurosurveillance. 2018;23:1700496. 